\title{
Which interventions are effective in preventing falls in people with multiple sclerosis? A Cochrane Review summary with commentary
}

\author{
Bhasker Amatya ${ }^{\mathrm{a}, \mathrm{b}, *}$ and Fary Khan ${ }^{\mathrm{a}, \mathrm{b}}$ \\ ${ }^{a}$ Department of Rehabilitation and Australian Rehabilitation Research Centre, Royal Melbourne Hospital, \\ Parkville, VIC, Australia \\ ${ }^{\mathrm{b}}$ Department of Medicine (Royal Melbourne Hospital), The University of Melbourne, Parkville, VIC, Australia
}

\begin{abstract}
. perspective. falls. robust studies are needed.

Keywords: Multiple sclerosis, falls, rehabilitation

The aim of this commentary is to discuss the rehabilitation perspective in the published Cochrane Review "Interventions for preventing falls in people with multiple sclerosis" (Hayes et al., 2019) by Hayes S, Galvin R, Kennedy C, Finlayson M, McGuigan C,
\end{abstract}

BACKGROUND: Falls are common and serious health concern in people with multiple sclerosis (MS). Various types of falls prevention interventions are currently investigated in people with MS.

OBJECTIVE: To assess the effectiveness of interventions to reduce falls in people with MS.

METHODS: To summarize falls prevention interventions from the published Cochrane Review "Interventions for preventing falls in people with multiple sclerosis" conducted by Hayes et al. Best available evidence was discussed from the rehabilitation

RESULTS: Overall 13 RCTs with 839 participants were included. The interventions evaluated included: exercise, education, and functional electrical stimulation alone or in combination. Majority of the included studies demonstrated high risk of bias. The findings suggest that the evidence was uncertain regarding the effects of evaluated interventions on preventing or reducing

CONCLUSIONS: The evidence for any falls prevention interventions in people with MS is sparse and uncertain, and more

*Address for correspondence: Dr Bhasker Amatya, Department of Rehabilitation Medicine, Royal Melbourne Hospital, 34-54 Poplar Road Parkville, Melbourne VIC 3052, Australia. Tel.: +61 3 83872037; Fax: +61 3 83872222; E-mail: bhasker. amatya@mh.org.au.
Walsh CD, Coote $S^{1}$, under the direct supervision of Cochrane Multiple Sclerosis and Rare Diseases of

\footnotetext{
${ }^{1}$ This summary is based on a Cochrane Review previously published in the Cochrane Database of Systematic Reviews 2019, Issue 12, Art. No.: CD012475, DOI: 10.1002/14651858. CD012475.pub2 (see www.cochranelibrary.com for information). Cochrane Reviews are regularly updated as new evidence emerges and in response to feedback, and Cochrane Database of Systematic Reviews should be consulted for the most recent version of the review.

The views expressed in the summary with commentary are those of the Cochrane Corner authors and do not represent the Cochrane Library or Wiley.
} 
the CNS Group. This Cochrane Corner is produced in agreement with NeuroRehabilitation by Cochrane Rehabilitation.

\section{Background}

Multiple sclerosis (MS) is a common neurodegenerative disorder of the central nervous system affecting approximately 2.3 million people worldwide (Browne et al., 2014). In people with MS, falls are one of the most important issues, resulting in serious and wide-ranging consequences. It is reported that approximately $56 \%$ of people with MS have falls during their disease course, with almost 37\% having recurrent falls. Falls have significant impact on patients' activity curtailment (mobility, ability for self-care and everyday living activities) and can contribute to physiological deconditioning, loss of independence, and institutionalization. Further, falls can have adverse impact on fear of falling and falls self-efficacy, and overall health (Hayes at al., 2019). These have significant economic implications and burden to the patients, carers/family, community and healthcare system in large (Sherrington et al., 2019).

The effectiveness of several interventions to minimize the risk-factors to prevent falls and associated injuries (including personal and environmental factors) have been reported in older adults living in the community (Sherrington et al., 2019). On the contrary, current published clinical guidelines do not outline an evidence-based approach to falls intervention, despite the high prevalence in people with MS. Hence, a recent Cochrane Review searched for evidence of the effectiveness of interventions preventing falls in people with MS (Hayes at al., 2019), aiming to guide the treating clinicians.

\section{Interventions for preventing falls in people with multiple sclerosis}

(Hayes S, Galvin R, Kennedy C, Finlayson M, McGuigan C, Walsh CD, Coote S. 2019)

\section{What is the aim of this Cochrane Review?}

The aim of this Cochrane Review was to evaluate the effectiveness of different types of falls prevention interventions in people with MS.

\section{What was studied and methods}

The falls prevention interventions considered in this review was any programme in which the primary or secondary aim was to prevent or reduce falls based on the taxonomy developed by the Prevention of Falls Network Europe (ProFANE). The ProFaNE taxonomy classifies all falls prevention as single interventions (one intervention component, such as exercise), multiple interventions (combination of two or more intervention components, such as exercise plus psychological interventions) or multifactorial interventions (participants receive different combinations of interventions based on an individual assessment of potential risk factors for falls).

The population addressed in this review was adults with clinically definite MS (all types). The intervention was compared to controls (wait-list control, usual care) or another type of falls prevention intervention/s. The primary outcomes studied included: falls rate (number of falls per person year), risk of falling (number of fallers) and adverse events. Secondary outcomes evaluated were: physiological falls risk, quality of life, balance function, cognition, MS disease progression, mobility, activity of daily living, self-reported fatigue and participation, assessed using specific scales.

\section{Search methodology and up-to-dateness of the Cochrane Review}

A comprehensive search of the literature was performed for randomized controlled trials (RCTs) and quasi-randomized trials using Cochrane Multiple Sclerosis and Rare Diseases of the CNS Group, Cochrane Central Register of Controlled Trials (2018 Issue 9), and prominent health science databases and clinical trials registries published up to 12 September 2018.

\section{What are the main results of the Cochrane Review?}

The review included 13 RCTs with 839 participants [mostly women, mean age 52 years (36 to 62 years)]. Interventions to reduce falls varied across included studies, the majority of which evaluated different types of exercise aimed to promote improvements in strength and balance function as a single intervention, or in combination with educational 
intervention. Most of the exercise programmes lasted from 6 to 12 weeks, with frequency of interventions ranging from once weekly to five times per week. Other interventions investigated were educational interventions and functional electrical stimulation (FES, common peroneal nerve stimulation). The active interventions were delivered in the community setting (group-based) or at home. They were compared either with no intervention (wait-list controls) or with another type of falls prevention intervention. Most included studies were of poor quality demonstrating high risk of bias related to one or more aspects of their methodology.

The key findings of the review are summarized below.

- Effectiveness of exercise interventions versus control

- very low-quality evidence for falls rate [rate ratio $(\mathrm{RaR})=0.68 ; 95 \%$ CI 0.43 to 1.06 ]

- low-quality evidence for number of fallers [risk ratio $(\mathrm{RR})=0.85 ; 95 \% \mathrm{CI} 0.51$ to 1.43 ]

- low-quality evidence for adverse events (RR of 1.25 ; $95 \%$ CI 0.26 to 6.03 )

- some beneficial effect for balance function [standardized mean difference $(\mathrm{SMD})=0.50$; $95 \%$ CI 0.09 to 0.92]; for self-reported mobility $(\mathrm{SMD}=16.30 ; 95 \% \mathrm{CI} 9.34$ to 23.26$)$ and objective mobility $(\mathrm{SMD}=0.28 ; 95 \%$ CI 0.07 to 0.50 )

- no evidence for improvement of other secondary outcomes such as physiological falls risk, fatigue, walking tests of mobility/walking endurance, and cognition

- No evidence of an effect in favor of educational interventions, exercise plus education, yoga or FES over control interventions for any of the falls outcomes

- For the comparison of other different falls prevention interventions, the heterogeneity of intervention types across studies prohibited the pooling of data

- No reported intervention-related adverse events

- No data on cost effectiveness of evaluated interventions

\section{How did the authors conclude on the evidence?}

The authors concluded that evidence regarding the effects of interventions for preventing falls in people with MS is sparse and uncertain. There is some evidence in favor of exercise interventions for improvement of balance function and mobility. However, this must be interpreted with caution as these secondary outcomes were not assessed under the GRADE criteria and results represent data from a small number of studies. The authors expressed the need for robust RCTs examining the effectiveness of multifactorial falls interventions on falls outcomes in MS population.

\section{What are the implications of the Cochrane evidence for practice in rehabilitation?}

Falls are common in MS and various rehabilitation interventions are currently trialled to reduce falls rate in this patient population. This review evaluated the effectiveness of interventions for preventing falls in people with MS, which included exercise, education, and functional electrical stimulation. The evidence in support of these interventions is limited and does not currently demonstrate significant positive results for falls outcomes. The findings suggest some evidence of the effect in favor of exercise interventions for improving balance function and mobility outcomes in MS. The evaluated falls prevention interventions appear to be safe.

\section{Acknowledgments}

The authors thank Cochrane Rehabilitation and Cochrane Multiple Sclerosis and Rare Diseases of the CNS Group for reviewing the contents of the Cochrane Corner.

\section{Conflict of interest}

The authors declare no conflicts of interest.

\section{References}

Browne, P., Chandraratna, D., Angood, C., Tremlett, H., Baker, C., Taylor, B. V., \& Thompson A. J. (2014) Atlas of multiple sclerosis 2013: a growing global problem with widespread inequity. Neurol (83), 1022-1024. DOI: 10.1212/WNL.0000000000000768 
Hayes, S., Galvin, R., Kennedy, C., Finlayson, M., McGuigan, C., Walsh, C.D., \& Coote, S. (2019) Interventions for preventing falls in people with multiple sclerosis. Cochrane Database Systc Rev (12). Art. No.: CD012475. DOI: 10.1002/14651858.CD012475.pub2
Sherrington, C., Fairhall, N. J., Wallbank, G. K., Tiedemann, A., Michaleff, Z. A., Howard, K., Clemson, L., Hopewell, S., \& Lamb, S. E. (2019) Exercise for preventing falls in older people living in the community. Cochrane Database Systc Rev (1). Art. No.:CD012424. DOI: 10.1002/14651858.CD012424.pub2 